OPEN ACCESS

Edited by:

Dangyuan Lei,

City University of Hong Kong, China

Reviewed by:

Tianyue Zhang,

Jinan University, China

Jian Ye,

Shanghai Jiao Tong University, China

${ }^{*}$ Correspondence:

Zhengkun Fu

zkfu@snnu.edu.cn

Zhenglong Zhang

zlzhang@snnu.edu.cn

${ }^{+}$These authors have contributed equally to this work

Specialty section: This article was submitted to Nanoscience,

a section of the journal

Frontiers in Chemistry

Received: 23 April 2021 Accepted: 17 June 2021

Published: 07 July 2021

Citation:

Mi X, Zhang T, Zhang B, Ji M, Kang B, Kang $C$, Fu Z, Zhang $Z$ and Zheng $H$ (2021) Binary Surfactant-Mediated Tunable Nanotip Growth on Gold Nanoparticles and Applications in

Photothermal Catalysis.

Front. Chem. 9:699548.

doi: 10.3389/fchem.2021.699548

\section{Binary Surfactant-Mediated Tunable Nanotip Growth on Gold Nanoparticles and Applications in Photothermal Catalysis}

\author{
Xiaohu Mi ${ }^{\dagger}$, Tingting Zhang ${ }^{\dagger}$, Baobao Zhang, Min Ji, Bowen Kang, Chao Kang, Zhengkun Fu *, \\ Zhenglong Zhang * and Hairong Zheng
}

School of Physics and Information Technology, Shaanxi Normal University, Xi'an, China

Plasmonic nanostructures with sharp tips are widely used for optical signal enhancement because of their strong light-confining abilities. These structures have a wide range of potential applications, for example, in sensing, bioimaging, and surface-enhanced Raman scattering. Au nanoparticles, which are important plasmonic materials with high photothermal conversion efficiencies in the visible to near-infrared region, have contributed greatly to the development of photothermal catalysis. However, the existing methods for synthesizing nanostructures with tips need the assistance of poly(vinylpyrrolidone), thiols, or biomolecules. This greatly hinders signal detection because of stubborn residues. Here, we propose an efficient binary surfactant-mediated method for controlling nanotip growth on Au nanoparticle surfaces. This avoids the effects of surfactants and can be used with other $\mathrm{Au}$ nanostructures. The Au architecture tip growth process can be controlled well by adjusting the ratio of hexadecyltrimethylammonium bromide to hexadecyltrimethylammonium chloride. This is due to the different levels of attraction between $\mathrm{Br}^{-} / \mathrm{Cl}^{-}$and $\mathrm{Au}^{3+}$ ions. The surface-enhanced Raman scattering and catalytic abilities of the synthesized nanoparticles with tips were evaluated by electromagnetic simulation and photothermal catalysis experiments (with 4-nitrothiophenol). The results show good potential for use in surface-enhanced Raman scattering applications. This method provides a new strategy for designing plasmonic photothermal nanostructures for chemical and biological applications.

Keywords: nanotip growth, binary surfactants, surface plasmon resonance, photothermal effect, SERS

\section{INTRODUCTION}

Plasmonic nanoparticles (NPs) are promising materials for nanoscale light confinement and manipulation, and have been widely used in identification (Liu et al., 2017; Qin et al., 2017; Ma et al., 2020; Mi et al., 2021), bioimaging (Mi et al., 2019; Wu et al., 2019; Falahati et al., 2020; Barella et al., 2021), and catalysis (Zhang et al., 2016; Zhang et al., 2019; Gelle et al., 2020). Because of their stable chemical properties, strong absorption, and wide-range tunable surface plasmon resonance (SPR) in the visible to near-infrared range (Huang et al., 2014; Ruan et al., 2014; Li et al., 2015; Sanchez-Iglesias et al., 2017; Wang et al., 2018), Au NPs show strong photothermal and electromagnetic (EM) field enhancements. These have important roles in optical signal 


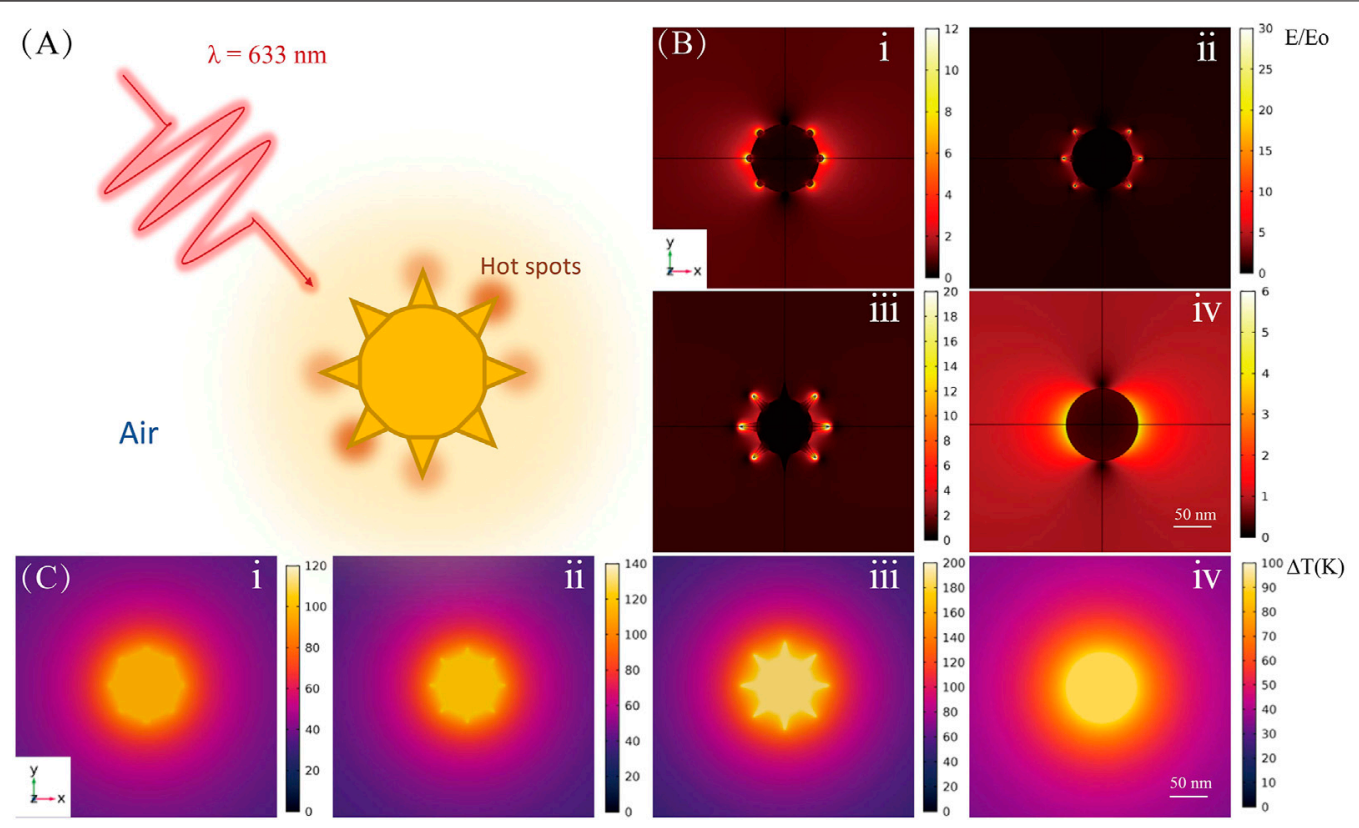

FIGURE 1 | Theoretical calculations for Au NP tips of different shapes. (A) Schematic diagram of laser-excited Au NP. (B) Electromagnetic field and (C) temperature distributions for Au nanoarchitectures; i, ii, iii, and iv correspond to the images in Figure 2Dii, iv, v, and viii, respectively.

enhancement applications (Wei and Xu, 2013; Ding et al., 2019; Langer et al., 2020; Yin et al., 2020). Plasmonic structures with sharp edges or tips can enhance an EM field near the edge or tip area 1000-fold because of charge aggregation, and they can minimize the field confinement to several nanometers (Schröder et al., 2015; Bombail et al., 2019). This strong field enhancement by structures with nanotips provides a new approach to the precision control and detection of optical signals, and can improve the efficiency of surface-enhanced Raman scattering (SERS) (Zhang et al., 2019). The strong light-absorbing ability of Au NPs not only gives strong EM field enhancement but also has great potential for improving photothermal conversion. This has already been widely used in photocatalysis (Shi et al., 2019) (Zhou et al., 2016; Zhang et al., 2019; Kong et al., 2020; Kong et al., 2021; Mateo et al., 2021).

In the traditional method for synthesizing plasmonic photothermal nanostructures with nanotips, poly(vinylpyrrolidone), thiols, and biomolecules are used as surfactants (Barbosa et al., 2010; Liu et al., 2014; Fan et al., 2018; Shi et al., 2020). However, these surfactants are hard to remove and can greatly affect the veracity of the measured optical signal, especially in Raman signal detection (Ansar et al., 2013; Zhang et al., 2020a; Zhang et al., 2020b; Qu et al., 2021). In addition, previous methods only enable the synthesis of specific structures with nanotips, for example, nanospheres, and the basic structure cannot be arbitrarily changed. It is therefore important to develop simpler methods for synthesizing nanostructures with controllable nanotips, which avoid the effects of surfactants.

In this study, we developed an efficient route for nanotip growth on Au nanostructures at room temperature. Importantly, we also solved the problem of surfactant removal by using a binary surfactant, that is, hexadecyltrimethylammonium chloride/hexadecyltrimethylammonium bromide (CTAC/ CTAB) instead of poly(vinylpyrrolidone), thiols, or biomolecules. Various $\mathrm{Au}$ nanoarchitectures were selectively synthesized by adjusting the CTAC/CTAB concentrations. Our synthetic method is simpler than the commonly used seedmediated growth method. Furthermore, the $\mathrm{Au}$ nanoarchitecture nanotips give better EM and photothermal field enhancements because the light confinement is stronger. This method provides a new strategy for designing plasmonic nanostructures.

\section{EXPERIMENTAL}

Chloroauric acid $\left(\mathrm{HAuCl}_{4} \cdot 4 \mathrm{H}_{2} \mathrm{O}\right), \mathrm{CTAB}, \mathrm{CTAC}, \mathrm{NaBH}_{4}$, $\mathrm{AgNO}_{3}$, and 4-nitrothiophenol (4-NTP, 80\%) were purchased from Sigma-Aldrich. $\mathrm{HCl}$, sodium citrate, ascorbic acid, $\mathrm{H}_{2} \mathrm{SO}_{4}$ (98\%), and $\mathrm{H}_{2} \mathrm{O}_{2}(50 \%)$ were purchased from the Sinopharm Chemical Reagent Co., Ltd. (China). Deionized water was used in all experiments.

Au NPs were synthesized by using a wet-chemical method that has been described in detail in our previous publications (Han et al., 2016). $\mathrm{HAuCl}_{4} \cdot 4 \mathrm{H}_{2} \mathrm{O}(0.01 \mathrm{M}, 2 \mathrm{ml})$ was dissolved in boiled deionized water $(78 \mathrm{ml})$ under stirring. Sodium citrate $(0.1 \mathrm{M}$, $8 \mathrm{ml}$ ) was quickly added to the solution. The mixture was kept at $100^{\circ} \mathrm{C}$ for $15 \mathrm{~min}$ under continuous stirring. The synthesized NPs were washed and centrifuged several times and then dispersed in water. They were stored in the dark at room temperature.

In a typical synthesis of other Au nanoarchitectures, $\mathrm{HAuCl}_{4}$ $(0.01 \mathrm{M}, 1 \mathrm{ml})$ aqueous solution was added to a CTAC/CTAB 

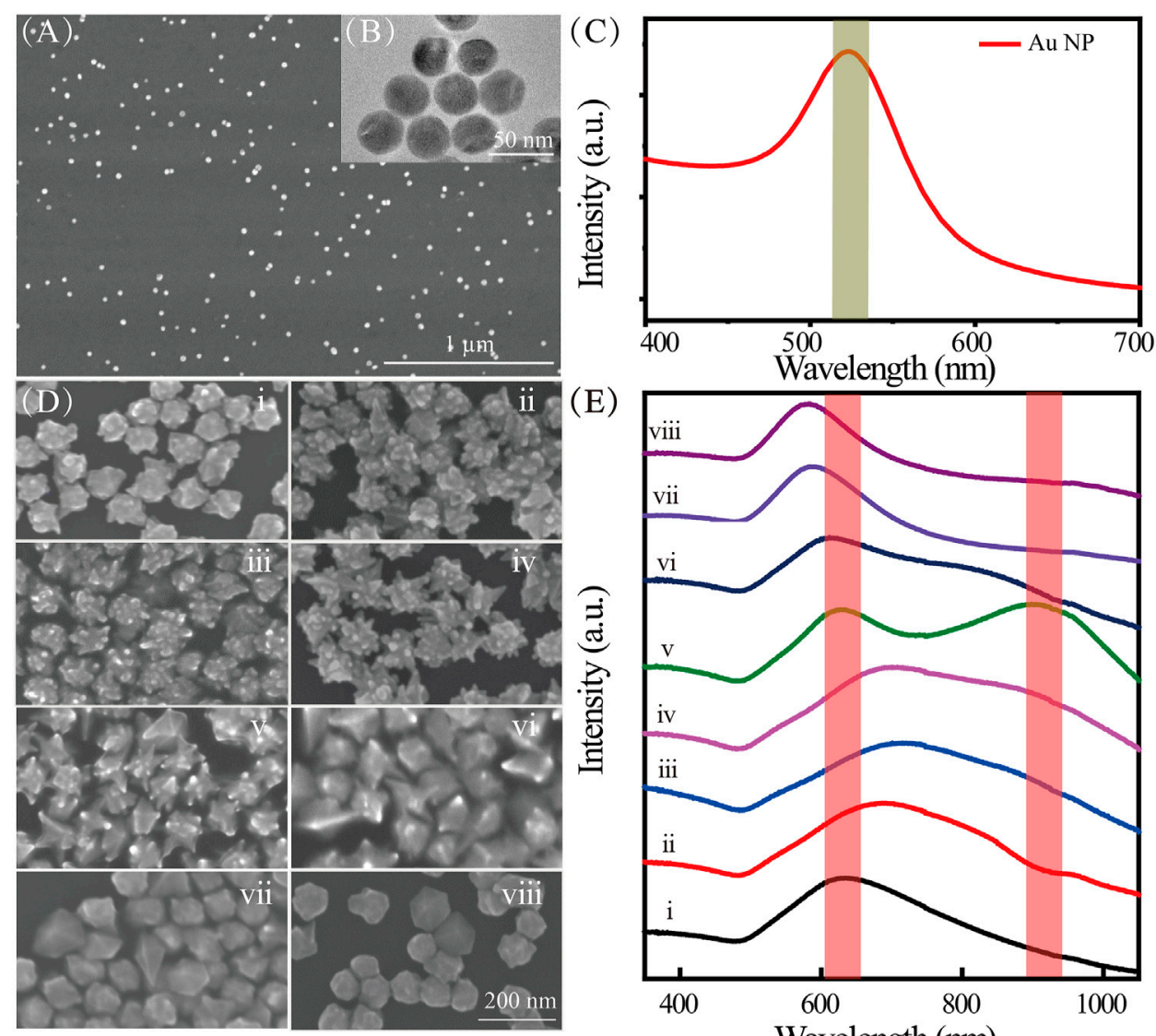

(E)

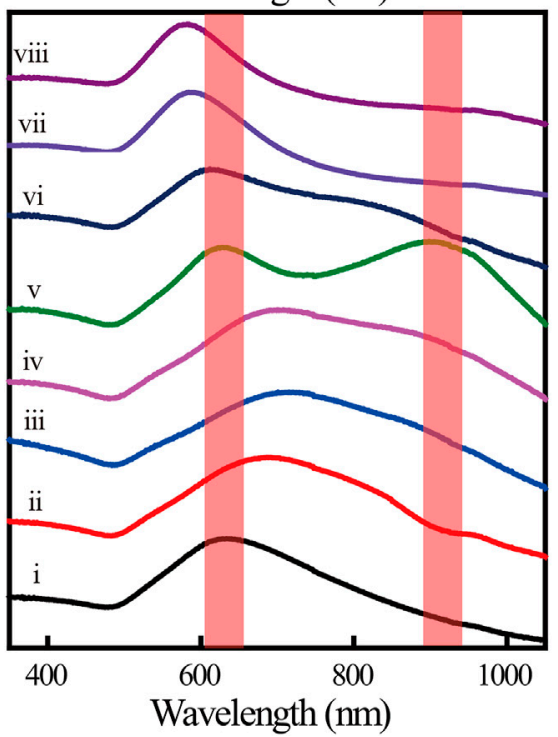

FIGURE 2 | (A) SEM and (B) TEM images of Au NPs; (C) extinction spectra of Au NPs; (D) SEM images of Au nanoarchitectures synthesized with different concentrations of CTAC and CTAB; (E) extinction spectra of Au nanoarchitectures synthesized with different concentrations of CTAC and CTAB. i-viii correspond to different concentrations (mM) of CTAC and CTAB, namely, 100/0, 98.75/1.25, 96.9/3.1, 87.5/12.5, 62.5/37.5, 50/50, 12.5/87.5, and 0/100, respectively.

$(0.1 \mathrm{M}, 20 \mathrm{ml})$ aqueous solution, and the mixture was vigorously stirred for $2 \mathrm{~min}$ to obtain a homogeneous solution. Then ascorbic acid $(0.1 \mathrm{M}, 0.16 \mathrm{ml})$ aqueous solution, $\mathrm{HCl}(0.2 \mathrm{M}$, $0.2 \mathrm{ml})$, and $\mathrm{Au} \mathrm{NPs}(200 \mu \mathrm{M})$ were added, and the mixture was stirred vigorously for $2 \mathrm{~min}$. The mixture was then left undisturbed at room temperature for $2 \mathrm{~h}$. The suspension was centrifuged at $3,000 \mathrm{rpm}$ for $5 \mathrm{~min}$, and the precipitate was collected, washed three times with ultrapure deionized water, and redispersed in deionized water $(2 \mathrm{ml})$.

$\mathrm{H}_{2} \mathrm{SO}_{4} / \mathrm{H}_{2} \mathrm{O}_{2}$ solution was prepared by mixing $\mathrm{H}_{2} \mathrm{SO}_{4}(12 \mathrm{ml})$ and $\mathrm{H}_{2} \mathrm{O}_{2}(28 \mathrm{ml})$. A silicon substrate was immersed in the mixed $\mathrm{H}_{2} \mathrm{SO}_{4} / \mathrm{H}_{2} \mathrm{O}_{2}$ solution at $80^{\circ} \mathrm{C}$ for $2 \mathrm{~h}$, and then washed with ethanol and deionized water. The Au nanoarchitectural species $(200 \mu \mathrm{L})$ were added to 4 -NTP $\left(10^{-7} \mathrm{M}, 4 \mathrm{ml}\right)$. The silicon substrate was placed in the solution at $30^{\circ} \mathrm{C}$ for $12 \mathrm{~h}$. The substrate was then washed with ethanol for $1 \mathrm{~min}$ and dried with highly pure $\mathrm{N}_{2}$ gas.

The samples were characterized by scanning electron microscopy (SEM) and transmission electron microscopy (TEM). The SEM images were obtained with an FEI-Nova Nano SEM 450 instrument at an operating voltage of $10 \mathrm{kV}$. The TEM images were obtained with a JEOL 2100 instrument at an accelerating voltage of $200 \mathrm{kV}$. Extinction spectra were recorded with a Perkin Elmer Lambda 950 spectrometer. All Raman and SERS spectra were obtained by using a Horiba Jobin Yvon LabRAM HR-Evolution Raman microscope with a $\times 100$ objective (Olympus, $\mathrm{NA}=0.9$ ) and 1800 grooves $\mathrm{mm}^{-1}$ grating. The excitation wavelength was $633 \mathrm{~nm}$. Each Raman spectrum was recorded with an accumulation time of $5 \mathrm{~s}$.

The finite element method (FEM) was used to perform numerical simulations of the EM field distribution. The electric and temperature field distributions were calculated by using the FEM; COMSOL software was used. The $633 \mathrm{~nm}$ incident light was shone from the $z$-direction and polarized in the $x$-direction. The average mesh size was set at $12 \mathrm{~nm}$. The radius and tip size of the $\mathrm{Au}$ nanostructures were set in accordance with the experimental results shown in Figure 1.

\section{RESULTS AND DISCUSSION}

As shown in Figure 2, the synthesized Au NPs were characterized by SEM (Figure 2A) and TEM (Figure 2B). They have uniform morphologies and good dispersity. The average diameter of the 

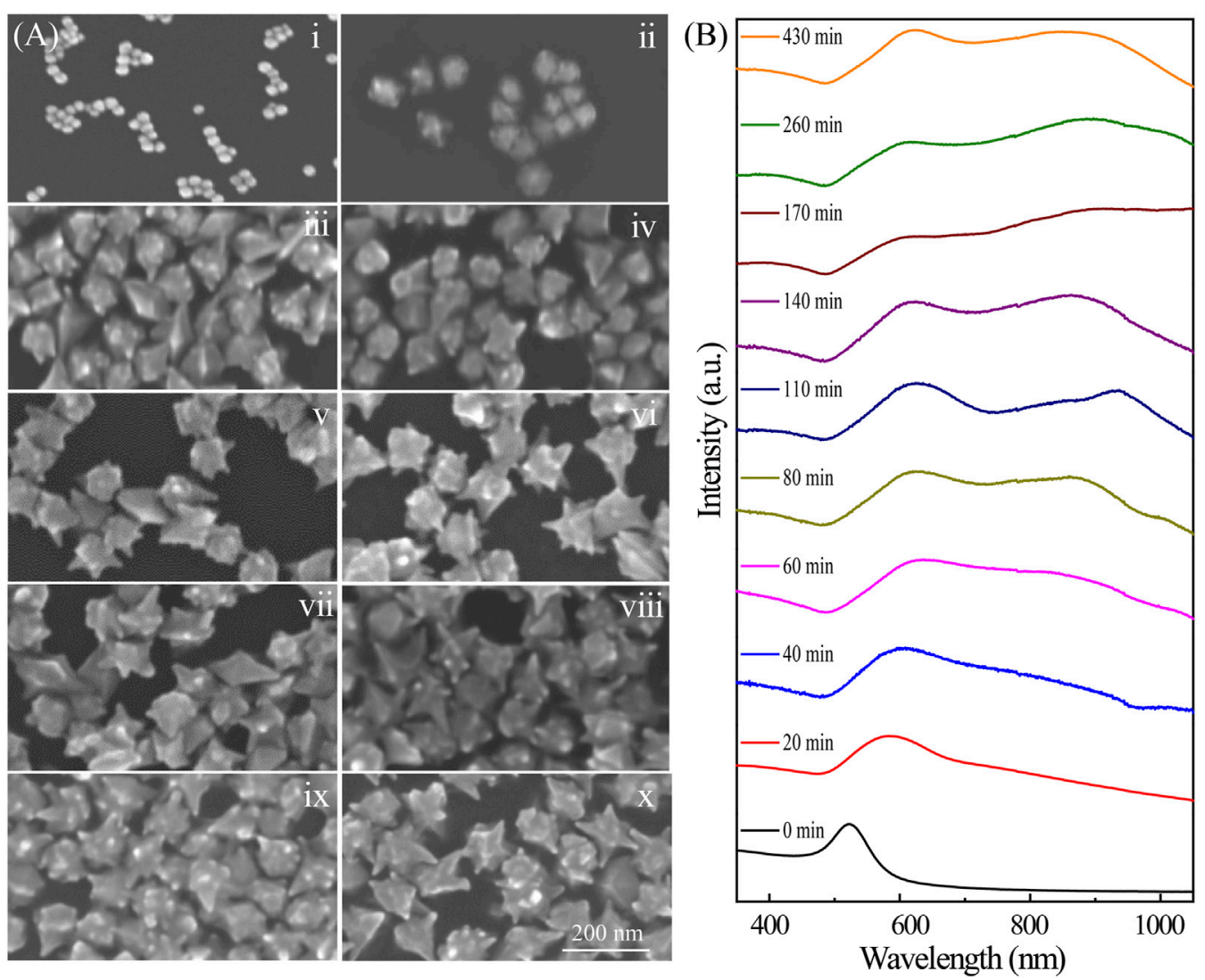

FIGURE 3 | (A) SEM images of Au nanoarchitectures synthesized with $0.2 \mathrm{mM} \mathrm{AgNO}_{3}$ for different reaction times. i-x correspond to 0, 20, 40, 60, 80, 110, 140, 170, 260, and $430 \mathrm{~min}$, respectively. (B) Extinction spectra of Au nanoarchitectures synthesized with $0.2 \mathrm{mM} \mathrm{AgNO}_{3}$ for different reaction times.

$\mathrm{Au}$ NPs is $30 \mathrm{~nm}$, which results in a distinct SPR peak at $\sim 520 \mathrm{~nm}$ (Figure 2C). The Au nanoarchitecture can be well controlled by adjusting the concentrations of CTAC and CTAB in the synthesis solution (Supplementary Figures S1, S2). The TEM image of representative nanotip from $\mathrm{Au}$ nanoarchitecture is shown in Supplementary Figure S2I. The Au NP morphology gradually changed with an increasing CTAB/CTAC ratio, from meatballlike shapes (Figure 2Di, no CTAB) to star-like (Figure 2Dii-vii) shapes, and to spherical-like shapes (Figure 2Dviii, no CTAC). The evolution of the Au NP morphology shows that $\mathrm{Br}^{-}$and $\mathrm{Cl}^{-}$ ions play important roles in the growth process. The Au NP evolution is also reflected by the extinction spectra shown in Figure 2E. As the $\mathrm{Br}^{-} / \mathrm{Cl}^{-}$ratio increases, a new SPR peak appears at approximately $900 \mathrm{~nm}$ (Figure 2Ev). This new peak appears because of the lightning-rod effect, in which the nanotip serves as an antenna. Coupling of the core and nanotip increases the effective dipole moment of the tip plasmons (Maity et al., 2014; Kim and Ha, 2017). Hybridization of the core and tip increases the cross section for excitation of bonding nanostar plasmons compared with that of an individual nanosphere plasmon.

Further insights into the growth mechanisms of the Au nanoarchitectures were obtained by using SEM to monitor the morphological changes with time, that is, after $0,20,40,60,80$,
110, 140, 170, 260, and 430 min (Figure 3). The Au NPs shown in Figure 2Dv, which were synthesized with CTAC/CTAB concentrations of $62.5 / 37.5 \mathrm{mM}$, are discussed in detail. The NP morphology distinctly changed with increasing time, namely, from meatball-like to branched and flower-like shapes. This indicates that the size and morphology of the Au crystals can be changed by controlling the chemical reaction time. The growth process involved in the NP evolution can also be deduced from the extinction spectra shown in Figure 3B. The meatball-like NPs observed at a reaction time of 20 min give a single SPR peak at $571 \mathrm{~nm}$. With increasing time, the SPR peak red-shifts to $630 \mathrm{~nm}$ (20-40 min) because of the increased particle size. After particle growth for $60 \mathrm{~min}$, a new SPR peak appears at $630 \mathrm{~nm}$. The corresponding absorption band broadens with increasing time to $160 \mathrm{~min}$. The peak beyond $800 \mathrm{~nm}$ in the extinction spectra of the Au nanoarchitectures (20-170 min) gradually appeared and redshifted because tip growth caused an increase in the effective dipole moment. At reaction times of $170 \mathrm{~min}$ and longer, the absorption spectra barely changed, which indicates that no further NP growth occurred. In a typical synthetic procedure, the products usually need to age for at least $3 \mathrm{~h}$ to obtain stable $\mathrm{Au}$ nanoarchitectures. Here, we describe a new method with a much shorter reaction time, which effectively improves the synthetic efficiency. 


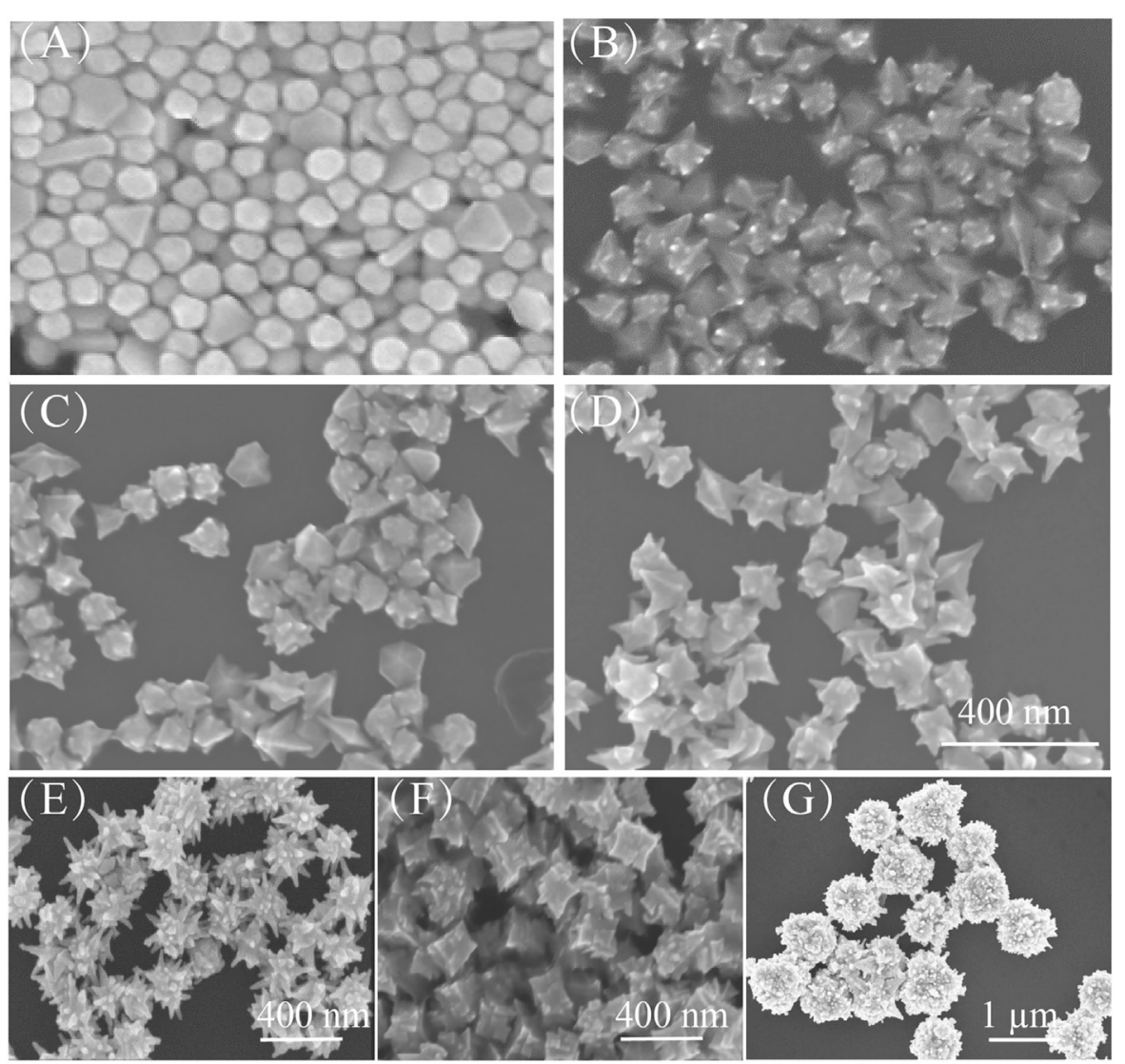

FIGURE 4 | (A-D) SEM images of Au nanoarchitectures synthesized with different concentrations of AgNO $: 0$ mM, 2 mM, 4 mM, and 8 mM. Scale bar is 400 nm. (E-G) SEM images of Au nanoarchitectures obtained with different seeds: (E) Ag NPs, (F) Au nanorods, and (G) Au nanoplates.

The growth of Au nanoarchitectures can also be affected by the concentration of $\mathrm{Ag}$ ions. Here, $\mathrm{Ag}$ ions at various concentrations, that is, $0 \mathrm{mM}$ (Figure 4A), $2 \mathrm{mM}$ (Figure 4B), $4 \mathrm{mM}$ (Figure 4C), and $8 \mathrm{mM}$ (Figure 4D), were used. As shown in Figures 4A-D, when no Ag ions were added, the Au NPs had no sharp points. However, the Au nanoflower tips became larger with increasing Ag ion concentration.

Figure 5 shows the growth of Au NPs with tips in detail. When $\mathrm{HAuCl}_{4}$ is mixed with CTAC and CTAB, $\mathrm{AuCl}_{4}$ ions bond strongly with $\mathrm{CTA}^{+}$, and complexes are formed (Khan et al., 2015). On addition of ascorbic acid, the $\mathrm{AuCl}_{4}-\mathrm{CTA}$ complex is partially transformed into $\mathrm{AuCl}-\mathrm{CTA}$; $\mathrm{Au}$ atoms are steadily released with time, and $\mathrm{HAuCl}_{4}$ is gradually reduced. The addition of $\mathrm{Ag}$ atoms from $\mathrm{AgNO}_{3}$ effectively modifies the $\mathrm{Au}$ $\mathrm{NP}$ surface (step i). Then $\mathrm{Cl}^{-}$ions are released and adsorbed at $\mathrm{Ag}$ sites to form strong $\mathrm{Ag}-\mathrm{Cl}$ bonds (step ii). Because of the high $\mathrm{Ag}-\mathrm{Cl}$ binding energy, $\mathrm{Au}$ atom migration to, and deposition at, these sites is inhibited. This leads to the formation of abundant nanotips as secondary structures on the Au NP surfaces (step iii) (Fan et al., 2018; Shi et al., 2020). The Au nanoarchitecture can easily be regulated by adjusting the concentration of $\mathrm{Cl}^{-}$ions. This nanotip growth method is suitable for Au NPs of other shape, for example, Ag NPs, Au nanorods, and Au nanoplates (Figures 4E-G).
For nanostructures with sharp tips, a strong electric field can be generated in the tip area because of a high aggregation of free charges; the LSPR decay can lead to a rapid and localized thermal effect, which provides the activation energy for surface chemical reactions. We simulated the EM and photothermal enhancement properties of NPs of various shapes. The enhancement effects for NPs with tips were evaluated by performing plasmon-driven catalytic reactions.

Figure 1 shows the simulation results for the EM field and temperature distributions for NPs with various shapes. The shapes correspond to different reaction times for tip growth on Au NPs. Figure 1A shows a schematic diagram of laserexcited Au NPs; the wavelength of the incident light is $633 \mathrm{~nm}$, and the particle is mediated in air. Figure 1B clearly shows that with tip growth, the electric field near the tip area is enhanced at least 30 -fold times at an excitation power of $1 \mathrm{~mW}$. This enhancement is five times larger than that for bare nanospheres. Tip growth not only contributes to stronger electric field enhancement but also improves the absorption ability of the NPs (i.e., increases the absorption cross section), which results in highly efficient photothermal conversion. As shown in Figure 1C, for bare NPs, the temperature rise under illumination is only $\sim 90 \mathrm{~K}$. However, with tip growth, the average NP temperature can 


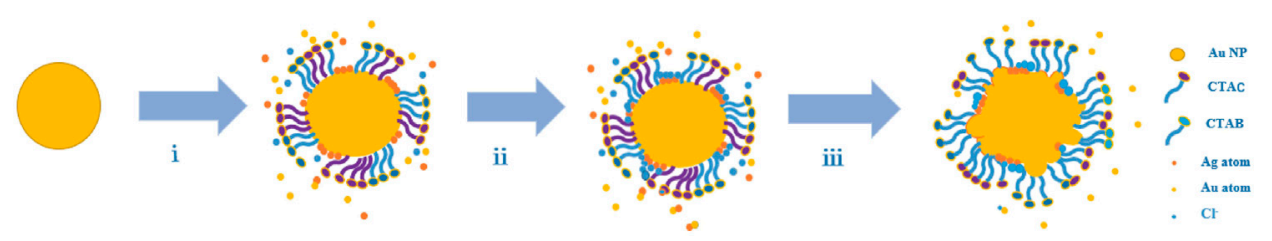

FIGURE $\mathbf{5}$ | Schematic diagram of growth of proposed Au nanoarchitectures.
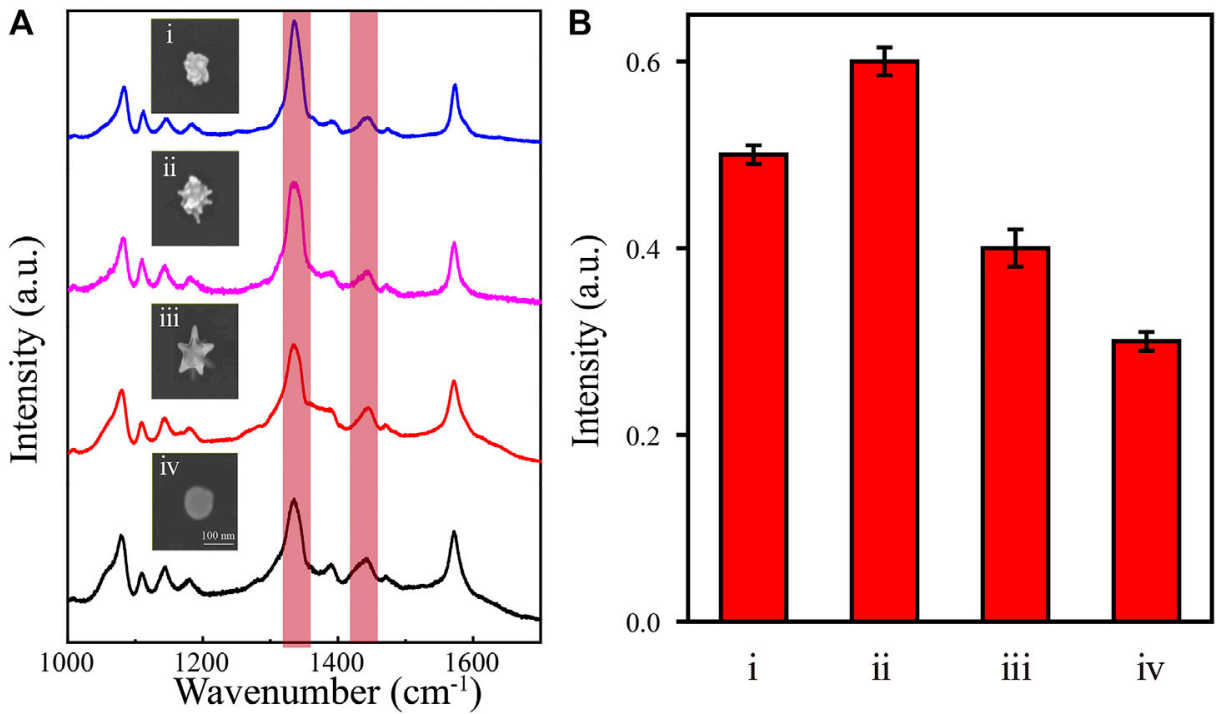

FIGURE 6 | (A) SERS spectra of 4-NTP $\left(10^{-7} \mathrm{M}\right)$ and SEM images of different Au nanoarchitectures. (B) The intensity ratio of $\left(1,435 \mathrm{~cm}^{-1} v_{\mathrm{N}=\mathrm{N}} / 1,335 \mathrm{~cm}^{-1} v_{\mathrm{NO} 2}\right)$ for different Au nanoarchitectures; i, ii, iii, and iv correspond to the images in Figure 2Dii, iv, v, and viii, respectively.

increase by $\sim 186 \mathrm{~K}$, which results in a better platform for photocatalytic reactions.

In a typical plasmon-catalyzed reaction, 4-NTP adsorbed on plasmonic nanostructures reacts exclusively to give $4,4^{\prime}$ dimercaptoazobenzene (DMAB) (Xie et al., 2013; Zhang et al., 2013; Kim et al., 2014). The plasmon-catalyzed dimerization of 4NTP to DMAB is characterized by the disappearance of the Raman band at $1,335 \mathrm{~cm}^{-1}\left(v_{\mathrm{NO} 2}\right)$ and the appearance of new Raman bands at approximately $1,140\left(\beta_{\mathrm{C}-\mathrm{H}}\right), 1,387\left(v_{\mathrm{NN}}+v_{\mathrm{CC}}+v_{\mathrm{C}-\mathrm{N}}\right)$, and $1,435\left(v_{\mathrm{N}=\mathrm{N}}\right) \mathrm{cm}^{-1}$ (Xie et al., 2011). The temperature is important in this chemical reaction. We used 4-NTP as a probe molecule to verify the photothermal effect of the synthesized NPs with nanotips. The SERS spectra from 4-NTP with various Au nanoarchitectures were recorded under $633 \mathrm{~nm}$ laser excitation at a power of $1 \mathrm{~mW}$ (Figure 6A). The results show that a new Raman peak appears at $1,435 \mathrm{~cm}^{-1}$, although the peak at $1,335 \mathrm{~cm}^{-1}$ is still strong. These features indicate that the catalytic reaction was successfully activated and the 4-NPT molecules were converted to DMAB. As shown in Figure 6B, the photothermal properties of NPs of various shapes were evaluated from the intensities of the peaks at 1,335 and $1,435 \mathrm{~cm}^{-1}$ in the SERS spectra. The results show that different $\mathrm{Au}$ nanoarchitectures have different photothermal properties. The meatball-like (Figure 3Aii) and star-like (Figure 3Aiv and v) samples are more efficient catalysts than NPs without any tips (Figure 3Aii). This is supported by the EM field and temperature distribution simulations shown in Figure 1. In summary, the photothermal properties of Au nanoarchitectures can be effectively adjusted and controlled by growth of different shapes.

\section{CONCLUSION}

A new efficient approach for growing nanotips on arbitrary $\mathrm{Au}$ nanoarchitectures was developed by simply adjusting the concentrations of CTAC and CTAB in the solution. The surface modification of AuNPs by AgCl leads to the formation of abundant nanotips as secondary structures. This method is not only suitable for growing nanotips on the surfaces of any metal NPs but also avoids surfactant effects. The EM and photothermal field enhancement effects of $\mathrm{Au}$ nanoarchitectures with nanotips are better than those achieved with architectures with smooth surfaces. This is because the nanotips lead to stronger light confinement. This was verified by FEM simulations and catalytic experiments. The simulation results show that the electric field enhancement with NPs with large tips is approximately five times that achieved with NPs without tips. In addition, the average temperature of the NPs gradually increases with tip growth because the absorption 
cross section increases. This provides a good platform for photothermal catalysis. This method provides a new approach to the design of highly efficient steady-state photothermal catalysts. It has potential applications in plasmonic sensors and photothermal treatment in biotechnology.

\section{DATA AVAILABILITY STATEMENT}

The original contributions presented in the study are included in the article/Supplementary Material; further inquiries can be directed to the corresponding authors.

\section{AUTHOR CONTRIBUTIONS}

$\mathrm{XM}$ and $\mathrm{TZ}$ conducted experimental work, did theoretical analysis, and drafted the manuscript; $\mathrm{BZ}, \mathrm{MJ}, \mathrm{BK}$, and $\mathrm{CK}$ proposed theoretical analysis. $\mathrm{ZF}$ and $\mathrm{ZZ}$ proposed the idea and joined in the preparation of the manuscript; $\mathrm{HZ}$ participated in the discussions on the experimental method and results.

\section{REFERENCES}

Ansar, S. M., Ameer, F. S., Hu, W., Zou, S., Pittman, C. U., Jr., and Zhang, D. (2013). Removal of Molecular Adsorbates on Gold Nanoparticles Using Sodium Borohydride in Water. Nano Lett. 13 (3), 1226-1229. doi:10.1021/ nl304703w

Barbosa, S., Agrawal, A., Rodríguez-Lorenzo, L., Pastoriza-Santos, I., Alvarez-Puebla, R. A., Kornowski, A., et al. (2010). Tuning Size and Sensing Properties in Colloidal Gold Nanostars. Langmuir 26 (18), 14943-14950. doi:10.1021/la102559e

Barella, M., Violi, I. L., Gargiulo, J., Martinez, L. P., Goschin, F., Guglielmotti, V., et al. (2021). In Situ Photothermal Response of Single Gold Nanoparticles through Hyperspectral Imaging Anti-stokes Thermometry. ACS Nano 15 (2), 2458-2467. doi:10.1021/acsnano.0c06185

Bombail, T., Garrigue, P., Goudeau, B., Talaga, D., Bouffier, L., Bonhommeau, S., et al. (2019). Dual Microelectrodes Decorated with Nanotip Arrays: Fabrication, Characterization and Spectroelectrochemical Sensing. Electrochimica Acta 328, 135105. doi:10.1016/j.electacta.2019.135105

Ding, S. J., Zhang, H., Yang, D. J., Qiu, Y. H., Nan, F., Yang, Z. J., et al. (2019). Magnetic Plasmon-Enhanced Second-Harmonic Generation on Colloidal Gold Nanocups. Nano Lett. 19 (3), 2005-2011. doi:10.1021/acs.nanolett.9b00020

Falahati, M., Attar, F., Sharifi, M., Saboury, A. A., Salihi, A., Aziz, F. M., et al. (2020). Gold Nanomaterials as Key Suppliers in Biological and Chemical Sensing, Catalysis, and Medicine. Biochim. Biophys. Acta (Bba) - Gen. Subjects 1864 (1), 129435. doi:10.1016/j.bbagen.2019.129435

Fan, Q., Liu, K., Feng, J., Wang, F., Liu, Z., Liu, M., et al. (2018). Building HighDensity Au-Ag Islands on Au Nanocrystals by Partial Surface Passivation. Adv. Funct. Mater. 28 (41), 1803199. doi:10.1002/adfm.201803199

Gellé, A., Jin, T., de la Garza, L., Price, G. D., Besteiro, L. V., and Moores, A. (2020). Applications of Plasmon-Enhanced Nanocatalysis to Organic Transformations. Chem. Rev. 120 (2), 986-1041. doi:10.1021/acs.chemrev.9b00187

Han, Q., Zhang, C., Gao, W., Han, Z., Liu, T., Li, C., et al. (2016). Ag-Au alloy Nanoparticles: Synthesis and In Situ Monitoring SERS of Plasmonic Catalysis. Sensors Actuators B: Chem. 231, 609-614. doi:10.1016/j.snb.2016.03.068

Huang, Y., Ferhan, A. R., Gao, Y., Dandapat, A., and Kim, D.-H. (2014). High-yield Synthesis of Triangular Gold Nanoplates with Improved Shape Uniformity, Tunable Edge Length and Thickness. Nanoscale 6 (12), 6496-6500. doi:10.1039/ c4nr00834k

Khan, M. N., Khan, T. A., Al-Thabaiti, S. A., and Khan, Z. (2015). Spectrophotometric Evidence to the Formation of AuCl4-CTA Complex and Synthesis of Gold Nano-

\section{FUNDING}

This work was supported by the National Key R\&D Program of China (Grant No. 2020YFA0211300), the National Natural Science Foundation of China (Grant Nos. 92050112, 12074237, and 12004233), and the Fundamental Research Funds for Central Universities (Grant Nos. GK202103010, GK202103018, and 2019TS118).

\section{ACKNOWLEDGMENTS}

The authors thank Professor Jiangbo Lu (Shaanxi Normal University) for the sample characterization by FEI Titan cubed Themis G2300 microscope.

\section{SUPPLEMENTARY MATERIAL}

The Supplementary Material for this article can be found online at: https:/www.frontiersin.org/articles/10.3389/fchem.2021.699548/ full\#supplementary-material

Flowers with Tailored Surface Textures. Spectrochimica Acta A: Mol. Biomol. Spectrosc. 149, 889-897. doi:10.1016/j.saa.2015.04.088

Kim, G. W., and Ha, J. W. (2017). Polarization-Sensitive Single Dipoles Generated from Multiple Sharp Branches on the Surfaces of Single Gold Nanourchins. J. Phys. Chem. C 121 (36), 19975-19982. doi:10.1021/acs.jpcc.7b06823

Kim, K., Choi, J.-Y., and Shin, K. S. (2014). Surface-Enhanced Raman Scattering of 4-Nitrobenzenethiol and 4-Aminobenzenethiol on Silver in Icy Environments at Liquid Nitrogen Temperature. J. Phys. Chem. C 118 (21), 11397-11403. doi:10.1021/jp5015115

Kong, T., Zhang, C., Gan, X., Xiao, F., Li, J., Fu, Z., et al. (2020). Fast Transformation of a Rare-Earth Doped Luminescent Sub-Microcrystal via Plasmonic Nanoislands. J. Mat. Chem. C 8 (13), 4338-4342. doi:10.1039/d0tc00060d

Kong, T., Zhang, C., Lu, J., Kang, B., Fu, Z., Li, J., et al. (2021). An Enhanced Plasmonic Photothermal Effect for Crystal Transformation by a Heat-Trapping Structure. Nanoscale 13 (8), 4585-4591. doi:10.1039/d0nr06714h

Langer, J., Jimenez de Aberasturi, D., Aizpurua, J., Alvarez-Puebla, R. A., Auguié, B., Baumberg, J. J., et al. (2020). Present and Future of Surface-Enhanced Raman Scattering. ACS Nano 14 (1), 28-117. doi:10.1021/acsnano.9b04224

Li, Q., Zhuo, X., Li, S., Ruan, Q., Xu, Q. H., and Wang, J. (2015). Production of Monodisperse Gold Nanobipyramids with Number Percentages Approaching $100 \%$ and Evaluation of Their Plasmonic Properties. Adv. Opt. Mater. 3 (6), 801-812. doi:10.1002/adom.201400505

Liu, Q., Yang, Y., Lv, X., Ding, Y., Zhang, Y., Jing, J., et al. (2017). One-step Synthesis of Uniform Nanoparticles of Porphyrin Functionalized Ceria with Promising Peroxidase Mimetics for $\mathrm{H} 2 \mathrm{O} 2$ and Glucose Colorimetric Detection. Sensors Actuators B: Chem. 240, 726-734. doi:10.1016/j.snb.2016.09.049

Liu, Z., Yang, Z., Peng, B., Cao, C., Zhang, C., You, H., et al. (2014). Highly Sensitive, Uniform, and Reproducible Surface-Enhanced Raman Spectroscopy from Hollow Au-Ag alloy Nanourchins. Adv. Mater. 26 (15), 2431-2439. doi:10.1002/adma.201305106

Ma, Y., Du, Y., Chen, Y., Gu, C., Jiang, T., Wei, G., et al. (2020). Intrinsic Raman Signal of Polymer Matrix Induced Quantitative Multiphase SERS Analysis Based on Stretched PDMS Film with Anchored Ag nanoparticles/Au Nanowires. Chem. Eng. J. 381, 122710. doi:10.1016/j.cej.2019.122710

Maity, A., Maiti, A., Das, P., Senapati, D., and Kumar Chini, T. (2014). Effect of Intertip Coupling on the Plasmonic Behavior of Individual Multitipped Gold Nanoflower. ACS Photon. 1 (12), 1290-1297. doi:10.1021/ph500309j

Mateo, D., Cerrillo, J. L., Durini, S., and Gascon, J. (2021). Fundamentals and Applications of Photo-Thermal Catalysis. Chem. Soc. Rev. 50 (3), 2173-2210. doi: $10.1039 / \mathrm{d} 0 \operatorname{cs} 00357 \mathrm{c}$ 
Mi, X., Wang, Y., Li, R., Sun, M., Zhang, Z., and Zheng, H. (2019). Multiple Surface Plasmon Resonances Enhanced Nonlinear Optical Microscopy. Nanophotonics 8 (3), 487-493. doi:10.1515/nanoph-2018-0231

Mi, X., Zhang, T., Zhang, C., Wang, Y., Chen, H., Li, J., et al. (2021). Plasmonic Sensing of Cu2+ via Shell-Etching of Au@Ag Nanorods. Mater. Chem. Phys. 259, 124036. doi:10.1016/j.matchemphys.2020.124036

Qin, L., Zeng, G., Lai, C., Huang, D., Zhang, C., Xu, P., et al. (2017). A Visual Application of Gold Nanoparticles: Simple, Reliable and Sensitive Detection of Kanamycin Based on Hydrogen-Bonding Recognition. Sensors Actuators B: Chem. 243, 946-954. doi:10.1016/j.snb.2016.12.086

Qu, Y., Wu, Z., Liu, Y., Lin, J., Zhang, L., and Luo, X. (2021). Impact of DoubleChain Surfactant Stabilizer on the Free Active Surface Sites of Gold Nanoparticles. Mol. Catal. 501, 111377. doi:10.1016/j.mcat.2020.111377

Ruan, Q., Shao, L., Shu, Y., Wang, J., and Wu, H. (2014). Growth of Monodisperse Gold Nanospheres with Diameters from $20 \mathrm{Nm}$ to $220 \mathrm{Nm}$ and Their Core/ Satellite Nanostructures. Adv. Opt. Mater. 2 (1), 65-73. doi:10.1002/ adom.201300359

Sánchez-Iglesias, A., Winckelmans, N., Altantzis, T., Bals, S., Grzelczak, M., and Liz-Marzán, L. M. (2017). High-Yield Seeded Growth of Monodisperse Pentatwinned Gold Nanoparticles through Thermally Induced Seed Twinning. J. Am. Chem. Soc. 139 (1), 107-110. doi:10.1021/jacs.6b12143

Schröder, B., Weber, T., Yalunin, S. V., Kiel, T., Matyssek, C., Sivis, M., et al. (2015). Real-space Imaging of Nanotip Plasmons Using Electron Energy Loss Spectroscopy. Phys. Rev. B 92 (8). doi:10.1103/PhysRevB.92.085411

Shi, F., He, J., Zhang, B., Peng, J., Ma, Y., Chen, W., et al. (2019). PlasmonicEnhanced Oxygen Reduction Reaction of Silver/Graphene Electrocatalysts. Nano Lett. 19 (2), 1371-1378. doi:10.1021/acs.nanolett.8b05053

Shi, Y., Li, Q., Zhang, Y., Wang, G., Matsuo, Y., Liang, X., et al. (2020). Hierarchical Growth of Au Nanograss with Intense Built-In Hotspots for Plasmonic Applications. J. Mater. Chem. C 8 (45), 16073-16082. doi:10.1039/d0tc04294c

Wang, Z., He, B., Xu, G., Wang, G., Wang, J., Feng, Y., et al. (2018). Transformable Masks for Colloidal Nanosynthesis. Nat. Commun. 9 (1), 563. doi:10.1038/ s41467-018-02958-x

Wei, H., and Xu, H. (2013). Hot Spots in Different Metal Nanostructures for Plasmon-Enhanced Raman Spectroscopy. Nanoscale 5 (22), 10794-10805. doi:10.1039/c3nr02924g

Wu, Y., Ali, M. R. K., Chen, K., Fang, N., and El-Sayed, M. A. (2019). Gold Nanoparticles in Biological Optical Imaging. Nano Today 24, 120-140. doi:10.1016/j.nantod.2018.12.006

Xie, W., Herrmann, C., Kömpe, K., Haase, M., and Schlücker, S. (2011). Synthesis of Bifunctional $\mathrm{Au} / \mathrm{Pt} / \mathrm{Au}$ Core/shell Nanoraspberries for In Situ SERS
Monitoring of Platinum-Catalyzed Reactions. J. Am. Chem. Soc. 133 (48), 19302-19305. doi:10.1021/ja208298q

Xie, W., Walkenfort, B., and Schlücker, S. (2013). Label-free SERS Monitoring of Chemical Reactions Catalyzed by Small Gold Nanoparticles Using 3D Plasmonic Superstructures. J. Am. Chem. Soc. 135 (5), 1657-1660. doi:10.1021/ja309074a

Yin, H., Li, N., Si, Y., Zhang, H., Yang, B., and Wang, J. (2020). Gold Nanonails for Surface-Enhanced Infrared Absorption. Nanoscale Horiz. 5 (8), 1200-1212. doi:10.1039/d0nh00244e

Zhang, D., You, H., Zhang, L., and Fang, J. (2020a). Facile Surface Modification of Mesoporous Au Nanoparticles for Highly Sensitive SERS Detection. Anal. Chem. 92 (23), 15379-15387. doi:10.1021/acs.analchem.0c02781

Zhang, X., Liu, B., Hu, C., Chen, S., Liu, X., Liu, J., et al. (2020b). A Facile Method in Removal of PVP Ligands from Silver Nanowires for High Performance and Reusable SERS Substrate. Spectrochimica Acta Part A: Mol. Biomol. Spectrosc. 228, 117733. doi:10.1016/j.saa.2019.117733

Zhang, Y., Gu, Y., He, J., Thackray, B. D., and Ye, J. (2019). Ultrabright gapenhanced Raman Tags for High-Speed Bioimaging. Nat. Commun. 10 (1), 3905. doi:10.1038/s41467-019-11829-y

Zhang, Z., Fang, Y., Wang, W., Chen, L., and Sun, M. (2016). Propagating Surface Plasmon Polaritons: Towards Applications for Remote-Excitation Surface Catalytic Reactions. Adv. Sci. 3 (1), 1500215. doi:10.1002/advs.201500215

Zhang, Z., Sun, M., Ruan, P., Zheng, H., and Xu, H. (2013). Electric Field Gradient Quadrupole Raman Modes Observed in Plasmon-Driven Catalytic Reactions Revealed by HV-TERS. Nanoscale 5 (10), 4151-4155. doi:10.1039/ c3nr00966a

Zhou, L., Tan, Y., Wang, J., Xu, W., Yuan, Y., Cai, W., et al. (2016). 3D SelfAssembly of Aluminium Nanoparticles for Plasmon-Enhanced Solar Desalination. Nat. Photonics 10 (6), 393-398. doi:10.1038/nphoton.2016.75

Conflict of Interest: The authors declare that the research was conducted in the absence of any commercial or financial relationships that could be construed as a potential conflict of interest.

Copyright (C) 2021 Mi, Zhang, Zhang, Ji, Kang, Kang, Fu, Zhang and Zheng. This is an open-access article distributed under the terms of the Creative Commons Attribution License (CC BY). The use, distribution or reproduction in other forums is permitted, provided the original author(s) and the copyright owner(s) are credited and that the original publication in this journal is cited, in accordance with accepted academic practice. No use, distribution or reproduction is permitted which does not comply with these terms. 